\title{
Wolbachia and mtDNA diversity and distribution in palearctic Drosophila melanogaster populations
}

\author{
R. Bykov ${ }^{1 *}$, I. Mazunin ${ }^{2}$, M. Yudina ${ }^{1,3}$, Yu. Ilinsky ${ }^{1,2,3}$ \\ ${ }^{1}$ Institute of Cytology and Genetics SB RAS, Novosibirsk, Russia \\ ${ }^{2}$ Immanuel Kant Baltic Federal University, Kaliningrad, Russia \\ ${ }^{3}$ Novosibirsk State University, Novosibirsk, Russia \\ *e-mail:bykovra@bonet.nsc.ru
}

Key words: Wolbachia, Drosophila melanogaster, mtDNA

\begin{abstract}
Motivation and Aim: Wolbachia symbionts are found in Drosophila melanogaster populations all over the world $[1,2]$. Genetic diversity of the symbiont in $D$. melanogaster is subdivided into several clades that have strong association with certain host mtDNA clades [3, 4]. Here we represent results of comprehensive surveys on cytoplasmic inheritance (Wolbachia and mtDNA) of fruit fly populations in a vast Palearctic territory. We address to the symbiont prevalence and symbiont genetic pattern as well as mtDNA pattern.

Methods and Algorithms: In total 1550 D. melanogaster samples were collected from different Palearctic localities. Samples were screened by PCR for Wolbachia infection and mitochondrial haplotypes.
\end{abstract}

Results: Wolbachia infection was found in every studied D. melanogaster populations from Western Europe to Far East. The average rate of infection was 0.56 (95\% confidence interval, 0.54-0.59). Infection rates are not dependent on longitude or latitude. Five mtDNA clades were found, where two of them (III and V clades) were predominant, that confirmed with previous data: the III clade is widely distributed in the world while the $\mathrm{V}$ clade is found only in Palearctic region.

Conclusion: We demonstrate wide Wolbachia distribution in Palearctic D. melanogaster populations. According to our data on symbiont diversity and distribution the fly populations of many regions in temperate zone renew after cold season. High frequency of the $\mathrm{V}$ clade of mtDNA in Palearctic populations may be explained by it having originated in this region.

Acknowledgements: Supported by the RFBR (16-04-00980).

\section{References}

1. Solignac M. et al. (1994) Widespread occurence of the proteobacteria Wolbachia and partial cytoplasmic incompatibility in Drosophila melanogaster. Comptes rendus de l'Académie des sciences. Série 3. Sciences Vie. 317(5):461-470.

2. Riegler M. et al. (2005) Evidence for a global Wolbachia replacement in Drosophila melanogaster. Current Biology. 15(15):1428-1433.

3. Richardson M.F. et al. (2012) Population genomics of the Wolbachia endosymbiont in Drosophila melanogaster. PLoS Genetics. 8(12):e1003129.

4. Ilinsky Yu. (2013) Coevolution of Drosophila melanogaster mtDNA and Wolbachia genotypes. PLoS One. 8(1):e54373. 